\title{
Sobre lo político y la política en jóvenes candidatos a diputaciones en elecciones presidenciales 2018
}

Recibido: 4 de octubre 2019

Revisado: 20 de febrero 2020

Aprobado: 16 de marzo 2020

Roy González Sancho

Costarricense. Licenciado en

Psicología y Máster en

Estudios Latinoamericanos

con énfasis en cultura y

desarrollo de la Universidad

Nacional, Costa Rica.

Investigador en el Centro

Agenda Joven en Derechos y

Ciudadanía de la

Universidad Estatal a

Distancia, Costa Rica.

Correo electrónico

rgonzalezs@uned.ac.cr

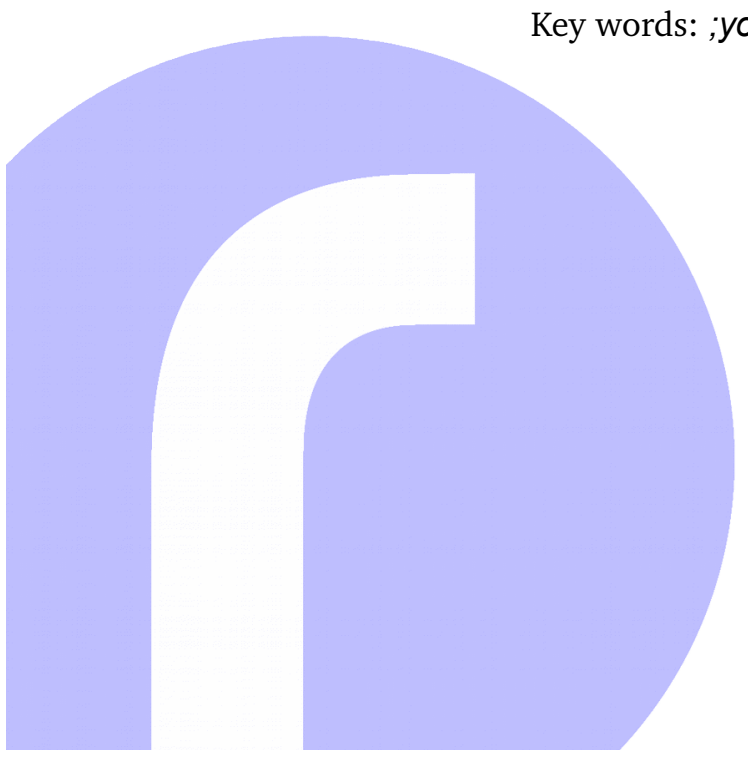

https://revistas.uned.ac.cr/index.php/rupturas (c) (i)(2)
Resumen: Este artículo se deriva de una investigación realizada en el Observatorio Político Electoral Juvenil (OPEJ) del Centro Agenda Joven de la UNED. Su objetivo es analizar las nociones de la política y las percepciones de la política en 7 personas candidatas a diputaciones en las elecciones de febrero de 2018. Parte de un enfoque cualitativo, con un alcance interpretativo y sigue la estructura de un ensayo científico. Algunos de los resultados indican diferencias entre las posturas externadas por las personas entrevistadas derivadas del tipo de ingreso a la participación política, las posibilidades de acción y las restricciones dentro de las estructuras partidarias.

Palabras clave: joven; política; participación juvenil; ciencias políticas; representación política

\section{On the Political and the Politics in Youth Candidates to the Congress in} Presidential elections 2018

Abstract: This article departs from a research made in the Observatorio Político Electoral Juvenil (OPEJ) at the Centro Agenda Joven UNED. Its aims to analyze notions of the policy and the perceptions of the policy in 7 candidates for deputies in the February 2018 Elections. It is based on a qualitative approach, with an interpretative scope and following the structure of a scientific essay. Some of the results indicate differences between the positions outsourced by the interviewed persons derived from the type of admission to political participation, the possibilities of action and restrictions inside the party structures.

Key words: ;youth; politics; youth participation; political science; democracy 
1. Dicho fallo de la CIDH obliga al Estado de Costa Rica hacer efectivo el reconocimiento todos los derechos que contempla la legislación vigente a las parejas del mismo sexo (Alfaro y Salazar 2018; Chinchilla y Cambronero 2018).

2. Uno de dichos efectos ha sido el reportado por Sancho (2018), quien publicó que, según una investigación del Centro de Investigación y

Promoción para América Central de Derechos Humanos (CIPAC), encontró que las personas costarricenses eran menos sensibles ante la comunidad LGBTI, luego de la campaña electoral de 2018 en comparación con 2013 , año en el que se realizó la medición anterior.

3. De acuerdo con el mismo autor, este hecho es remarcable, debido a que también corresponde a la primera vez en 18 años que una mujer preside el congreso y la tercera en toda la historia del país.

4. La composición pasó de tres mujeres y dos hombres en 2014 a ocho mujeres y cuatro hombres en 2018.

\section{Introducción:}

Las pasadas elecciones presidenciales y legislativas de 2018 se desarrollaron en medio de una campaña electoral caracterizada por un contexto agitado, debido a temas álgidos que ocuparon los titulares de los medios de comunicación de Costa Rica y las principales tendencias de "discusión" en las redes sociales en internet durante la primera y segunda vuelta.

Algunos de estos fenómenos fueron los discursos emitidos de ciertos candidatos presidenciales en temas como seguridad y empleo, así como el fallo de la Corte Interamericana de Derechos Humanos (CIDH) del 24 de noviembre de 2017 y publicado el 9 de enero de $2018^{1}$.

Ante las reacciones de la opinión pública, de acuerdo con Cascante Lizano (2018) y Alfaro (2018), instituciones, como la Defensoría de los Habitantes y la Universidad Nacional, emitieron comunicados, en los cuales llamaron al cese de las manifestaciones de odio, xenofobia y homofobia que se hicieron evidentes durante el desarrollo de la campaña, tanto en redes sociales como en reportes de agresiones físicas y verbales sufridas por varias personas. Las repercusiones de estas dinámicas incluso alcanzarían a verse luego de completado el proceso electoral ${ }^{2}$.

En medio de las condiciones anteriormente descritas, en la primera ronda electoral, se concretó la conformación definitiva de las Diputaciones de la Asamblea Legislativa para el periodo 2018-2022. Esta se caracteriza inicialmente por presentar, de acuerdo con Arrieta (2018), una menor edad promedio con respecto a la administración anterior y de contar, según Soto (2018), con un directorio legislativo con presidencia y vicepresidencia a cargo de dos mujeres jóvenes ${ }^{3}$, esto en su primer año. Por último, siguiendo a Artavia Medrano (2018) en este mismo inicio de periodo legislativo, se ha aumentado la cantidad de curules ocupadas por personas jóvenes, pasando de cinco en el periodo 2014-2018 a doce ${ }^{4}$ en el inicio de 2018-2022.

El trabajo acá presentado se desprende de la investigación realizada desde el Observatorio Político Electoral Juvenil (OPEJ) del centro Agenda Joven de la Universidad Estatal a Distancia (UNED), cuyo foco de atención es el estudio de las prácticas políticas que caracterizan el comportamiento electoral de las personas jóvenes los procesos electorales nacionales.

Ante las circunstancias detalladas, el objetivo principal de este ensayo científico, en particular, es analizar las nociones de lo político y las percepciones de la política que han construido, a partir de sus trayectorias político-partidarias, las personas jóvenes candidatas a diputaciones en las elecciones presidenciales de 2018.

Dicho propósito ha surgido debido a la variedad de usos, las posturas y el análisis de ciertas situaciones que las personas jóvenes candidatas han deja- 
do entrever en sus relatos acerca de sus trayectorias de participación política, comprendidas desde las formas de ingreso a la política formal hasta la culminación de la inscripción de sus candidaturas para las elecciones del 2018.

\section{Aspectos teóricos}

\section{Lo político y la política}

De previo a la escritura de este artículo, se realizó una breve búsqueda de los principales trabajos teóricos en torno a los siguientes conceptos clave: lo político y la política, la juventud y lo joven. Esto con la intención de ubicar la reflexión de este artículo en las coordenadas más apropiadas y acordes a las exigencias del problema de investigación, la metodología y el foco de análisis.

Sobre estos conceptos, se ha podido observar, a partir de la literatura consultada (Díaz Gómez 2003; Retamozo Benítez 2009; Mouffe 2011; Aguilar López 2011; Abarca 2012; Arias-Cardona y Alvarado 2015; Dussel 2016), que ambos términos suelen usarse en ocasiones de forma equivalente sin mayores diferenciaciones. No obstante, es posible establecer, conforme al propósito de este trabajo, una delimitación básica para el análisis del talante que adquieren ambos conceptos en las narraciones de las personas jóvenes entrevistadas desde las trayectorias.

De acuerdo con Retamozo Benítez (2009) y Mouffe (2011), lo político es una noción ontológica cuyo elemento constitutivo reside en el conflicto ${ }^{5}$, el cual comporta una relación de "confrontación" que sucede entre posiciones contrarias o antagónicas -de sujetos o conjuntos de estos-, y cuyos productos son las distintas configuraciones que conforman lo social ${ }^{6}$ (como las subjetividades, el género, la cultura, la economía, democracia, etc.).

La relevancia de este aspecto para lo político es tal, que, según Dussel (2016), lo social es donde reaccionan los actores implicados en sus dinámicas al tomar conciencia; por ejemplo, de sus reivindicaciones incumplidas, produciéndose así las crisis o los problemas sociales que se observan materializados en diversas formas, como acciones y manifestaciones de solidaridad, descontento, reclamo, etc.

Ante esta suerte de acontecimiento, nace la urgencia de resolver, de organizar y de delimitar el devenir de lo social por medio de cierto orden o que contenga estas dinámicas o relacionales en marcos de referencia o estructuras comunes. Así, Dussel (2016), Díaz Gómez (2003) y Mouffe (2011) coinciden en considerar que dicho medio es la política, la cual se conforma de aquellas prácticas, relaciones sociales sedimentadas e instituidas a partir de las que, inicialmente, se ordena u organiza la convivencia social, así como la canalización de las necesidades colectivas y las reivindicaciones sociales, tanto aquellas ya garantizadas como las emergentes.
5. De acuerdo con ambas fuentes, el conflicto ha sido un aspecto que el pensamiento político ha intentado cancelar mediante la conformación de instituciones que lo apacigüen y evitar, en consecuencia, reflexionar sobre este y construir a partir de las tensiones resultantes.

6. En este aspecto, tanto Dussel (2016) como Mouffe (2011) insisten que lo social se conforma de las relaciones antagónicas que mediante su repetición durante la historia, se han "sedimentado" dando forma distintas estructuras arquetípicas de relaciones que configuran o delimitan las interacciones sociales. No obstante, lejos de ser un proceso terminado, estas las relaciones sociales se mantienen en constante cambio por el conflicto entre quienes participan de esas interacciones. 
7. En el sentido de que, en tanto categoría, se define en relación con otras categorías como lo adulto, el género, la clase social, etc. (Pérez Islas 2000; Bourdieu 2002; Margulis y Urresti 2008)
Podría plantearse que, a grandes rasgos, la política es al mismo tiempo un medio práctico por el cual se logra organizar, distribuir o delegar el poder que marca el campo de acción de lo político y las partes implicadas en esta dinámica.

\section{La juventud y lo joven (joven)}

Respecto de las discusiones teóricas sobre la juventud, de acuerdo con diferentes personas investigadoras como Pérez Islas (2000), Aguilar López (2011), Arias-Cardona y Alvarado (2015) y Bendit y Miranda (2017), el estudio académico de dicho tema se ha realizado desde varios y diversos enfoques. Entre estos últimos, se encuentran estudios estructurales-funcionalistas; paradigmas culturalistas y estudios culturales; diferentes vertientes del marxismo; escuelas de la sociología francesa hasta llegar a espacios más institucionales de la administración estatal como el enfoque de juventudes y de desarrollo de políticas públicas.

De tal forma, los conceptos utilizados para referirse a la juventud y a lo joven, en palabras de Brito Lemus (1998), Pérez Islas (2000), Bendit y Miranda (2017) y Pico Merchán y Vanegas García (2014), pasaron de ser alusiones que nominalizaban a un individuo a estudiar la cuestión como un fenómeno social o colectivo, de carácter relacional ${ }^{7}$ e histórico.

Al tomar en cuenta lo anterior, cuando se menciona juventud en este trabajo, se parte de las posturas de expuestas por Brito Lemus (1998); Bourdieu (2002) y Villa Sepúlveda (2011), para quienes este concepto puede definirse como un entramado sistema de prohibiciones, de censuras o de limitaciones en relación con las acciones sociales que las personas pueden llevar a cabo. Además, deriva del estatus de subordinación al que son sometidas aquellas individualidades categorizadas como jóvenes respecto de aquellas definidas como adultas.

Así pues, la juventud se manifiesta como una expresión de la jerarquía u orden social generacional y como un resultado o producto de los procesos de dominación y de sujeción de unas generaciones sobre otras. Dicho de otra manera, siguiendo lo planteado por Bourdieu (2002), como derivación de la cuestión del poder, en cuanto a la repartición de este, del orden y espacios sociales en los que se supone "cada persona debe estar", conforme a la reproducción del orden social.

Esa dinámica, de acuerdo con Brito Lemus (1998), tiene su origen en la existencia de un saber adulto que parte de la experiencia acumulada por el tiempo vivido de la persona adulta, como ventaja ante otra que carece de este atributo y que, en razón de esto, se encuentra en una relación de dependencia. Entonces, la juventud sería un producto de aquello que, en este ensayo, se ha considerado como lo político, ya que resulta de relaciones antagónicas entre las generaciones en "conflicto".

De esta forma, lo joven podría establecerse, partiendo de los planteamientos de Margulis y Urresti (2008) y Villa Sepúlveda (2011), como ese espacio rela- 
cional más inmediato a la práctica, en el cual aquellas personas consideradas jóvenes participan mediante "nuevos modos" de la reproducción de lo social y establecen acciones, sentidos o significados contingentes ${ }^{8}$ al orden social establecido; o bien, generan propuestas o proyectos sociales que pueden, del mismo modo, perpetuar o contravenir las disposiciones marcadas por lo adulto y sus relaciones con las personas categorizadas o consideradas como tales.

Así, mientras la juventud se observa como el conjunto de circunscripciones de posibilidad y acción para quienes no poseen "experiencia vivida acumulada" respecto de las que sí cumplen este atributo, el espacio de lo joven se demarcaría por el modo de agenciar o de accionar. Con ello, las personas definidas socialmente como "jóvenes" se desempeñan en un medio determinado, dentro o fuera de las delimitaciones que lo adulto aplica o asigna a las relaciones con su contraparte "antagónica".

\section{Metodología}

Como se mencionó, el objetivo principal de este trabajo consistió en analizar las percepciones de la política y las nociones de lo político que las personas jóvenes, candidatas a Diputaciones en las elecciones presidenciales de 2018, han construido a partir de sus trayectorias político-partidarias.

Dicho propósito partió de un enfoque cualitativo, con un alcance interpretativo y sigue la estructura de un ensayo científico. De acuerdo con Jaramillo Ríos y Mendoza Martínez (2004), Guanipa Pérez (2011) e Hidalgo-Capitán (2012), tal ensayo es una producción académica en la que una persona investigadora -o varias- exponen, de forma sistemática y rigurosa, una postura subjetiva sobre un tema específico, con base en resultados de investigaciones científicas y especializadas propias o de terceros relativas al área de conocimiento desde la cual se desarrolla la discusión.

De esta forma, se estudian a fondo los resultados y las transcripciones literales de varias entrevistas en profundidad aplicadas a personas jóvenes candidatas a Diputaciones para las elecciones presidenciales del 2018, cuya intención fue analizar sus trayectorias de participación político-partidaria, llevadas a cabo en el marco del proceso de investigación del Observatorio Político Electoral Juvenil (OPEJ) del Centro Agenda Joven de la UNED.

Los datos y los resultados tomados como unidades de información para este trabajo se han producido mediante la técnica de investigación denominada entrevista en profundidad. Para Gaínza Veloso (2006) y Taylor y Bogdan (1987), esta técnica se caracteriza por tratarse de un encuentro o varios en los que quien los dirige no asume un papel directivo con respecto a quien se entrevista. En ella, se realizan preguntas que siguen más una guía flexible de temas o de puntos por tratar que una jerarquía u orden definido por la persona investigadora, cuyo foco de atención es la experiencia de la persona entrevistada antes que el interés del investigador o de la investigadora.

\author{
8. Es decir que pueden estar a \\ favor o en contra.
}


9. La distribución del grupo entrevistado fue la siguiente: de la provincia de Cartago, la joven candidata del Frente Amplio (FA) y el candidato del Partido Unidad Social Cristiana (PUSC); de la provincia de San José, las candidatas de los partidos Alianza Demócrata Cristiana (ADC), Partido de los Trabajadores (PT) y Acción Ciudadana (PAC); de la provincia de Alajuela, la joven candidata del PUSC; y de la provincia de Puntarenas, el candidato del Partido Liberación Nacional (PLN).
En dicho instrumento, se planteó una guía de diez puntos nodales sobre las trayectorias de participación partidaria del grupo entrevistado. Contempló los siguientes aspectos: detalles biográficos; primeras experiencias de participación política, labores y puestos ocupados en dichas instancias; procesos de formación política; percepciones sobre la juventud en general, sobre la sociedad en general de ellas y ellos como jóvenes políticos; situación de las personas jóvenes en la política en general y dentro de sus partidos, etc.

Así, partiendo de las experiencias narradas en las transcripciones literales de las entrevistas y de los resultados de su sistematización, en este artículo, se analiza las nociones de lo político y las percepciones de la política que han construido siete personas jóvenes candidatas a Diputaciones en las elecciones presidenciales de febrero de 2018.

Las transcripciones analizadas corresponden a las entrevistas realizadas a cinco mujeres y a dos hombres menores de 35 años, provenientes de seis partidos políticos de cuatro de las siete provincias de Costa Rica ${ }^{9}$. Se llevaron a cabo, entre el 29 de enero y el 6 de marzo de 2018, así como el informe final de los resultados entregado en el Centro Agenda Joven agosto del mismo año por González-Sancho (2018).

Para la escogencia de las unidades de análisis estudiadas en este ensayo científico, se siguieron los siguientes criterios: 1. Experiencia de, al menos, seis años de trayectoria política. 2. Claridad en las posiciones políticas expuestas. 3. Descripción detallada de sus roles y actividades en las estructuras partidarias en las que participaron.

Se excluyeron los casos de las personas jóvenes candidatas que no cumplieran las disposiciones mencionadas. No obstante, en este caso, al igual que en el informe de investigación original de González-Sancho (2018), la procedencia geográfica de las personas entrevistadas no fue un impedimento para su inclusión, ya que se ha trabajado con quienes pudieron ser contactadas en medio del desarrollo de la primera y la segunda vuelta de las elecciones presidenciales de 2018.

Por último, para el análisis de los documentos, se utilizó la categorización, la cual, partiendo de los planteamientos de Taylor y Bogdan (1987) y Cisterna Cabrera (2005), consiste en seleccionar, organizar y dar significado a la información en estudio a partir de categorías de análisis previas -apriorísticas- 0 emergentes en el proceso de escrutinio de datos. En este ensayo científico, se ha analizado la información desde dos categorías apriorísticas, a saber: noción de lo político y percepción de la política. 


\section{Resultados}

\section{Algunos datos preliminares, grupo familiar y participación política}

Previo al análisis de las nociones de lo político y las percepciones de la política, se hace necesaria una introducción breve sobre la procedencia de quienes brindaron los datos e información estudiados en este trabajo.

Inicialmente, se destaca que todas las personas jóvenes entrevistadas provienen de familias cuyos progenitores poseen grados de educación universitaria y una situación socioeconómica estable. Al mismo tiempo, huelga decir que el grupo de jóvenes cuenta con estudios superiores, en disciplinas de ciencias sociales, ciencias exactas y de administración, con grados que van desde el bachillerato hasta la maestría.

También, la participación y la filiación política han sido elementos predominantes en diversas formas en los entornos familiares, al punto que los padres de las personas entrevistadas del PAC, del ADC, del PUSC y del PLN resultaron electos en puestos de gobiernos locales y partidarios. Para el caso de las jóvenes del FA y del PT, familiares cercanos fueron quienes militaron directamente con los partidos políticos o eran simpatizantes activos de estos en los procesos electorales.

Mientras tanto, las personas jóvenes entrevistadas del PLN, del PUSC y del ADC manifestaron que, junto con sus familiares, participaron activamente como militantes de los partidos desde edades muy tempranas. A diferencia de estas, las jóvenes del PT, del FA y del PAC detallaron que su ingreso a las estructuras político-partidarias sucedió luego de estar involucradas en espacios de participación comunal, acciones colectivas y movimientos sociales.

Así, de los datos proporcionados por el conjunto de jóvenes entrevistados, puede observarse que provienen de grupos familiares en los cuales los procesos de electorales locales y nacionales no se han restringido a votar únicamente, sino que parece tratarse de una tradición significativa dentro de este grupo primario, acorde con el clima político y educativo recién detallado. Sin embargo, como se verá en el análisis siguiente, las percepciones de la política y las nociones de lo político presentan contrastes considerables.

\section{Nociones de lo político}

Este apartado condensa lo que en este artículo se ha denominado como lo político, el cual remite al componente simbólico o no tangible que estructura, de diversas formas, las relaciones que han vivenciado las personas jóvenes a través de su participación dentro de las estructuras políticas. De este modo, se han rescatado las referencias clave con las cuales las personas candidatas nominalizaban un "nosotros" joven en diálogo o negociación, que disputa o está en concordancia con lo adulto.

En este sentido, algunas posturas del conjunto entrevistado revisten puntos valiosos de cómo esta atribución se define respecto al otro en los procesos 
de ingreso a la política. En ocasiones, encarnado en una persona adulta; en otras, por jóvenes.

Aquí se denotaron diferencias entre las personas con antecedentes partidarios fuertes y aquellas cuyo ingreso a la política tuvo que ver con otros eventos externos al grupo familiar. En el caso de la primera, la entrevistada del PUSC, describía de la siguiente forma el contexto en el que entraba a dicho partido político:

... Bueno, yo nací social cristiana, además, ehh, mis papás son ambos de familias tradicionales social cristianas y mi mamá siempre ha sido una importante dirigente social cristiana en San Carlos... di, pues realmente toda, toda mi vida ha estado rodeada de política, desde, desde pequeña recuerdo, diay, pues, todas las elecciones mi mamá siempre trabajando y dirigiendo el partido en San Carlos, entonces, fue como parte también de, de, de mi formación del día a día... Siempre he militado en el partido; de hecho, he sido delegado distrital, cantonal y provincial desde mis dieciocho años, entonces, o sea por un tiempo tal vez no, no, no estuve en distritales entonces no fui delegada, pero a mis dieciocho años, fui por primera vez delegada, ehh, provincial inclusive del partido, entonces, diay, pues, he sido una militante desde nacimiento del Partido Unidad Social Cristiana... entonces, realmente no, no tengo un punto de inicio ni de escogencia... (p. 2)

En este caso, puede denotarse que hay una suerte de simetría o acuerdo tácito entre el sujeto que relata su ingreso a la política y las prácticas desempeñadas dentro de las estructuras partidarias, al punto que, con base en los mismos antecedentes que aporta la joven, parece ser su entrada a esta instancia un paso "natural" y sin conflictos dentro de un proceso vital descrito por ella. Algo similar ha sucedido con los dos varones entrevistados de partidos tradicionales (PUSC y del PLN). En este sentido, parece que ese "nosotros", detallado en la cita anterior, corresponde más con una posición acorde con lo adulto y su reproducción dentro del partido que un rol en disputa o diálogo. Esto debido a que, en ambos casos, los jóvenes no acotaron mayores discordancias con las estructuras y jerarquías de sus respectivos partidos. 
En cuanto a las otras jóvenes entrevistadas, se han hallado elaboraciones de esa instancia de referencia -"nosotros" o "yo"-, presentes igualmente en el ingreso al espacio de la vida partidaria, pero con matices diferentes. Así, la joven entrevistada del FA detalló sus primeros acercamientos de la siguiente manera:

...yo creo que yo ingreso a la política como tal, más o menos tenía como catorce años ...entonces en ese momento yo estaba involucrada mucho, tenía muchos amigos que patinaban, entonces esa fue como la discusión que tenía siempre, no, de que nunca había un espacio donde patinar, que la policía siempre les caía y entonces empezamos a hacer como un grupillo ahí a ver cómo nos organizábamos y a partir de eso fue como yo me empecé a involucrar como en lo más comunal, no y conseguimos donaciones, conseguimos varias cosillas verdad ...Con la gente del teatro, a pesar de que nunca hice teatro sí había una, toda una organización de organizaciones, era una red de organizaciones juveniles, que al final yo terminé, di, pues, trabajando en esa red y a partir del 2012 es que ya me involucro en la política partidaria (p. 6)

Como puede verse, su ingreso está marcado por un acercamiento desde la participación en el nivel local con agrupaciones y organizaciones cantonales de personas jóvenes, abocada a resolver, de forma colectiva, situaciones de necesidades inmediatas para la población joven del cantón. Algo similar acontece con las experiencias descritas en las trayectorias de las entrevistadas del PAC, del PT y, en menor medida, la joven del ADC. En este sentido, se observa que ese "nosotros" parece estar orientado, en estos casos, a la negociación y al desarrollo de propuestas para el abordaje de una situación concreta.

También, se denota cómo las personas jóvenes candidatas han reaccionado ante eventos trascendentales para la realidad nacional, tal cual fue la coyuntura del Referéndum para la aprobación de la agenda de implementación del Tratado de Libre Comercio entre Estados Unidos, Centroamérica y República Dominicana en 2006 y en 2007. Las entrevistadas del PT, del PAC, del FA y del ADC, quienes se refirieron a este hecho, se encontraban en estos momentos cursando sus estudios secundarios o universitarios, mientras que, en paralelo, estaban iniciando su involucramiento con espacios de participación política formal. 
Una primera aproximación de lo recién señalado puede verse en el relato de la joven entrevistada del ADC, quien describió la situación de la siguiente forma:

Bueno, yo nunca fui miembro activo del movimiento estudiantil... El movimiento estudiantil de la UCR es sumamente politizado; lamentablemente y en mi época, era la época del TLC... entonces, en mi época, usted estaba en contra del TLC o no existía y yo estaba a favor del TLC, entonces... el movimiento estudiantil de la UCR siempre ha estado a favor de los grupos de, de izquierda, entonces, para nadie eso es un secreto y, bueno, yo no, yo no profeso una ideología de izquierda, yo me ubico más en el centroderecha, entonces, nunca me sentí cómoda con el movimiento estudiantil de la Universidad de Costa Rica. Pero, sin embargo, sí participé en algunas luchas que tenían que ver, digamos, por problemas inmediatos que yo enfrentaba, como que el edificio se nos inundaba, como que el edificio estaba siempre lleno de palomas, como que el edificio el ascensor no servía con muchísima frecuencia. Entonces, ese fue más que todo mi rol en el movimiento estudiantil, recuerdo alguna vez participar en alguna marcha para la lucha por la paz o cosas de esa naturaleza cuando era estudiante de, de la Universidad y en el rol que teníamos en la radio, en el programa Tiempos de política donde hacíamos mucho análisis de coyuntura... mucho análisis de realidad tanto del movimiento estudiantil como de la como de la realidad nacional del país en, en general.

Partiendo de lo expresado por la joven, se puede visualizar que el "yo" político expuesto toma distancia de otros sujetos colectivos jóvenes en forma antagónica, no solo por las diferencias en torno al evento político que da contexto a su declaración, sino por las divergencias ideológicas e intereses políticos que deja manifiestos en su intervención. Al instante, también, es claro que su interés y rango de participación política consistía, en aquel entonces, 
en apoyar o en involucrarse en acciones o en manifestaciones que estuviesen orientadas a solucionar alguna necesidad o problema inmediato dentro de la comunidad universitaria.

Mientras tanto, para las jóvenes del PAC y del FA, la importancia que tuvo subjetivamente el proceso del referéndum para la implementación del TLC es diferente del testimonio anterior. La primera cuenta su experiencia desde un contexto urbano y del Valle Central. La segunda habla desde una región rural de un cantón fuera de la Gran Área Metropolitana (GAM). Respecto del caso de la joven candidata del PAC, esta detalló lo siguiente:

Ehh, yo sentía una inquietud, verdad, estábamos en la adolescencia, estaba empezando a negociarse, era la época de la negociación del TLC. Yo tenía muchas dudas, veníamos saliendo del Combo, entonces había mucha efervescencia social y en el colegio no me respondían esas preguntas. Cuando yo tomo esos libros empiezo a ver respuestas, empiezo a ver lo que es un movimiento social, de protesta, lo que es, este, ehmm, bueno este, organización de grupos, lo que son cuestionamientos al statu quo, etc... Y empiezo a encontrar muchas respuestas y, y a encontrar como una vocación de vida. Ahí empecé a meterme en un montón de grupos, ambientalistas y demás (joven candidata PAC, p. 3).

Mientras que la joven candidata del FA ha señalado lo siguiente:

En el colegio en donde yo estaba en ese momento era muy poca gente... porque estaba hasta noveno y habían, ehh, habían treinta y cinco personas en todo el colegio y de esos treinta y cinco, solo dos éramos del NO, entonces para mí sí, sí marca una discusión inclusive con mis mismos compañeros de clase; yo creo que ahí es donde yo empiezo como a tratar de involucrarme de alguna $u$ otra forma no, que, al final, termino siendo como de parte de la, la parte de los muchachos y muchachas que pati- 
naban, pero como una forma de tratar de cambiar algo no, de algo que no estaba bien (joven candidata FA, p. 7).

Las entrevistadas en este caso, en un sentido contrario al expuesto por la joven del ADC, narran cómo se demarca ese "nosotros", con la particularidad de que se antagoniza a partir de una necesidad de reflexionar y de actuar por cambios locales y nacionales. Especialmente, motivados a causa de la falta de respuestas a sus inquietudes sobre un tema coyuntural y a la ausencia de puntos comunes en sus instituciones educativas con las demás personas adultas y jóvenes que formaban parte de ellas.

Así, esa búsqueda por responder sus inquietudes, además de haber tenido un rol importante en el posicionamiento político de las jóvenes candidatas entrevistadas, frente al significado e implicaciones del TLC, abrió la posibilidad de conocer las estructuras de participación de mayor alcance, como movimientos sociales, agrupaciones comunales y partidos políticos en los que militaron o se involucraron.

El aspecto básico de coyunturas como esta yace en cómo manifiesta la diversidad de formas de "hacer sociedad". Necesariamente, estas últimas pasan por la confrontación, el surgimiento o la consolidación de subjetividades y de posicionamientos al respecto de este proceso, así como por las construcciones derivadas en prácticas, en discursos y en lineamientos colectivos e individuales.

Un aspecto que también llama la atención, en las trayectorias de participación política de dicho grupo de jóvenes candidatos, son las referencias sobre los apelativos o las censuras que han experimentado por su condición o atribución de juventud, de parte de las personas adultas del partido en las labores habituales, así como de gente externa en las actividades de campaña política.

El primer ejemplo de ello puede ser el adultocentrismo en las estructuras internas de los partidos. Consultado sobre las dificultades o barreras experimentadas en su curso a la candidatura, el joven candidato del PUSC afirmó lo siguiente:

Siempre hay resistencia a la juventud por definición, ni siquiera es porque me cae mal o es un odioso, es porque simplemente ahí hay gente que considera que uno no tiene la experiencia suficiente para, para afrontarlo y eso, eso, digamos, es lo, lo constante, ehh, y después de eso, es que también tenemos un problema comunicacional. Somos súper chapas a la hora de comunicar, ehh, y, no le sacamos provecho a las participaciones jóvenes que te- 
nemos, entonces, diay, tenemos hoy un secretario gene-

ral de treinta y tres años y no, el país no sabe, entonces

se... eso, eso es todo un tema verdad... (p. 6)

Esta intervención, además de evidenciar una actitud o una práctica adultocéntrica dentro del partido, expone parte de esas mediaciones desde las cuales se posiciona una persona joven en estos entornos con respecto del conflicto generacional. De acuerdo con los detalles brindados por el joven, ese conflicto parece estarse presentando como un problema de fondo dentro de la gestión de actividades del partido político. No obstante, también se destaca el distanciamiento que establece al momento de referirse sobre el problema de comunicación con las personas jóvenes dentro y fuera del partido, en el sentido de parecer un "yo" joven en acuerdo con lo adulto o, al menos, extraño al de sus semejantes.

Por su parte, la joven candidata del PAC ante la misma pregunta de las dificultades que afrontó en su proceso de candidatura advertía que, además del adultocentrismo, otro elemento presente en las dinámicas partidarias es el machismo, de manera que detalló lo siguiente:

El machismo ha sido lo, digamos, yo no, si si, estee... Por ejemplo, ahora que fui coordinadora del Plan de Gobierno lo viví, ehh, usualmente el Plan de Gobierno estaba en manos de, de un grupo de hombres, ehh, de ciertas edades y demás, entonces lo he vivido, digamos, pese a que yo soy oficialmente la coordinadora Plan de Gobierno en todo espacio me presentan como: "Ella es una muchachita que nos ayuda con el Plan de Gobierno", entonces, yo tengo que corregir y decir: "No, iyo soy la coordinadora del Plan de Gobierno!". Que no debería estar pasando, entonces digamos, me ven como muchachita a mí a los treinta y uno, no quiero imaginar a una chica de veinte, como, como la ven (p. 32).

De acuerdo con lo relatado por la joven candidata, esta situación tiene lugar, con mayor medida, en las designaciones y en los procesos internos informales del partido. Caso contrario al mostrado en los procesos de conformación de las papeletas presentadas en las elecciones nacionales, en las cuales se cumplen las cuotas de paridad de género, por ejemplo. 
De esta forma, cuando actividades informales, como la coordinación de tareas internas del partido o las exposiciones dentro de sus comisiones e instancias, son realizadas por una mujer joven, suelen ser habituales las desacreditaciones y los menosprecios por las labores efectuadas.

En un sentido similar, las jóvenes del PUSC, del FA y del ADC han manifestado que, con alguna frecuencia, han sido objeto, tanto dentro como fuera de sus partidos, de descalificaciones de sus capacidades; principalmente, por su condición de jóvenes y de mujeres. Esas descalificaciones se han dado de forma directa, pasivo-agresiva y en referencias en las redes sociales, en algunas ocasiones.

Una acotación brindada por el joven candidato del PLN ha dado un matiz adicional al tema de las confrontaciones o estructuras relacionales que enfrentan él y las demás personas jóvenes. Este corresponde con las formas en las que variaron sus posibilidades de participación en los procesos de formulación de propuestas o la toma de decisiones dentro del partido político una vez obtenida su candidatura. Al respecto, detalló lo siguiente:

Sí, en algún momento se, se dificultaba más porque uno tenía que utilizar otros medios, verdad. Había que utilizar a terceras personas para poder... "mirá, es que a mí me parece que esto debe ser así, así" y la persona: "Sí, a mí me parece que estás diciendo esto, pero me parece que habría que incluir otra cosa", entonces, el mensaje se iba a medias... Ehh, y ahora, pues, el asunto es diferente, verdad. Yo considero alguna opción, entonces, ya la, la planteo directamente. Sí, pero siempre anteriormente sí había, este, iguales situaciones, pero tenía que utilizar otros canales para poder hacer llegar el mensaje (p. 14).

En esta declaración, puede denotarse que haber conseguido la candidatura le significó un paso imprescindible que le concedió las facultades y los permisos necesarios para involucrarse directamente en las gestiones o en las planificaciones propias del partido político, atributos ausentes en los puestos ocupados por el joven con anterioridad. Dicho resultado puede ser interpretado como una investidura que lo ha ligado, casi de forma instantánea, a lo adulto, legitimando, al menos inicialmente, sus aportes y su participación en los procesos "serios" de la estructura partidaria -tomar decisiones, generar propuestas o proyectos-. Dichas potestades, en apariencia, son exclusivas de aquellas personas con mayor capital de experiencia acumulado dentro de la organización. 
Ante la misma experiencia, es decir, la investidura que significa lograr la Diputación en un puesto con posibilidades de elección, otras personas jóvenes entrevistadas han vivido resultados diferentes, a saber: las mujeres jóvenes. Estas, a pesar de contar con la investidura de candidatas a una Diputación en la papeleta de sus partidos, se han subestimado o han recibido descalificaciones sobre sus capacidades como lideresas o representantes de la organización; en dos de esos casos, aun siendo diputadas electas.

\section{Percepciones de la política}

En esta sección, se abordan las percepciones sobre las formas o las prácticas que se ejecutan desde los espacios institucionalizados para organizar, canalizar, reivindicar o resolver las necesidades, los conflictos y las exigencias que tanto sujetos particulares como colectivos plantean sobre la sociedad y sus instancias.

De esta forma, puede denotarse, inicialmente, que existe una diferencia palpable en la manera en la que el conjunto de entrevistados ha ingresado a sus respetivos partidos políticos. En el caso de las jóvenes candidatas del PAC, del FA y del PT, se ha destacado que, dentro de las instancias previas al partido, su participación política inició en organizaciones comunales o movimientos sociales, mientras que, para el colectivo de jóvenes del PUSC, del ADC y del PLN, esta etapa ha transcurrido desde instancias institucionales. Por ejemplo: la candidata del ADC y el candidato del PLN han puntualizado su paso por las asociaciones o los grupos de estudiantes en sus etapas de educación superior, en tanto que las dos personas jóvenes entrevistadas del PUSC han señalado que su ingreso fue directamente al partido político.

La anotación anterior ofrece un marco de referencia de las percepciones sobre la política que las personas jóvenes candidatas han utilizado para describir sus trayectorias de participación, así como las maneras en las que han intentado cumplir con sus objetivos particulares o colectivos.

En este sentido, algunas de las declaraciones de las personas candidatas con relación a cómo perciben el quehacer de la política son similares en algunos aspectos. En lo que respecta a las jóvenes entrevistadas del ADC y del PUSC, parte de sus declaraciones parece sugerir que conciben la política como una suerte de labor orientada al abordaje de las necesidades de la gente.

Una explicación de este punto se encuentra en la siguiente acotación emitida por la joven del ADC, al preguntársele por experiencias de participación adicionales a las vividas en el partido político que milita:

...hay una parte que a uno no se le puede olvidar como

politóloga y tiene que ver con la conexión con la realidad

social del país. Yo formo parte de algunas ONG, trabajo,

por ejemplo, como voluntaria en "Chepe se Baña",... he 
estado en diversos movimientos sociales, ehh, desde amarrarme en el edificio de Ciencias Sociales viejo de la UCR para exigir un edificio nuevo... hasta participar en procesos para buscar que, a la gente de Los Guido, de Desamparados, se les habilite matrícula y puedan sus hijos entrar a clases mañana. Entonces, siempre he tenido esa conexión y esas ganas de entender que la política es servicio y esas ganas de entender de que la política es un vehículo para mejorar la calidad de vida de la gente ( $p$. $6)$.

Como se aprecia en este fragmento, la preocupación de la entrevistada corresponde a dejar explícito que el objeto de la política y su actuar debería destinarse a mejorar la vida de las personas o a cubrir las urgencias de la población. Este punto es compartido por la entrevistada del PUSC, quien considera que su labor como diputada electa debe partir de un compromiso con toda la gente que votó por ella -aunque en realidad la votación se hace por el partido político-, quienes creyeron en ella para representarlos y resolver sus necesidades más apremiantes.

Ahora bien, en el caso de los varones del PUSC y del PLN, ambos candidatos electos insistieron en percepciones de política enfocadas al quehacer y a las labores partidarias, en un sentido que parece dirigirse a la gestión de las decisiones, las propuestas y los procesos de la instancia. De esta forma, la percepción del joven candidato del PUSC puede rastrearse siguiendo sus afirmaciones cuando se le preguntó sobre su mayor logro en su trayectoria de participación política:

Yo creo que sacar al partido de la crisis financiera, no solo yo, pero digamos, eh eh, creo que esa restructuración me parece que es un ejemplo, humm, difícilmente si, si no le hubiera dedicado el tiempo y, y las cosas, ah..., hubiéramos tenido estos resultados, me parece. Insisto no soy solo yo, es varia gente, pero digamos, yo he estado ahí tomando y recomendando, tomando decisiones y recomendando decisiones y creo que eso, eso es fundamental porque ya lo demás es personal, digamos, a nivel institucional es lo que vale verdaderamente, ehh..., es eso, tener al partido sano financieramente (p. 6). 
Así, puede denotarse, por la forma como ha detallado este hito personal dentro del partido, que la política concebida por el entrevistado trata de emprender o de liderar acciones de organización o de reestructuración de procesos del partido político, las cuales podrían estar basadas en el convencimiento, en la persuasión o en el diálogo con otras personas de la instancia para lograr dichos objetivos. No obstante, el mismo entrevistado ha reconocido que, en ocasiones, principalmente por un "bloqueo generacional" presente en quienes conformaban las estructuras del partido, tuvo que imponerse o ser más ejecutivo para abrirse espacio y posibilidades en este cometido.

Por su lado, el joven candidato del PLN ha dejado patente su percepción sobre la política al referirse a su trayectoria de participación en distintos espacios previos al partido político:

...por ejemplo, la Universidad de Costa Rica realizaba proyectos, eh, a lo interno de la, de la carrera, de la facultad, en los que uno... estaba entrando a la universidad y, pues, eso maneja cierto grado de negociación. Eh, a nivel de la carrera también hay cursos donde te, te obligan a tener que interactuar, a tener que promover, a tener que vender a tener una idea, y pues, eso al final y al cabo es la política verdad... y te hace ir formando $y, y, y$ vas participando. Yo creo que, sin lugar a dudas, incidió mucho, ehh, la forma, la carrera que escogí, ehh, y también, cómo se me enseñaba... (p. 4).

En este extracto, parece que el joven candidato concibe el quehacer de la política como un proceso de promoción o mercadeo de una idea o proyecto político, cuyo fin es convencer a las demás estructuras de una instancia para la consideración en agenda o implementación de la propuesta planeada. Sin embargo, no debe perderse de vista que este tipo de operación dentro de las estructuras partidarias puede ser funcional en el cabildeo o planteamiento de temas relevantes dentro de la misma dinámica institucional de la organización.

Ahora bien, en lo que respecta a la candidata del PAC, su percepción sobre la política se enfoca, en mayor medida, en un compromiso que debe mover a la juventud partidaria y al resto de la instancia hacia un contacto más amplio con sus coetáneos externos. La joven lo detalla de la siguiente forma:

...ha habido un compromiso, por ejemplo, de minimizar

cada vez más el peso de lo electoral en el partido político.

Yo sé que un partido político, al final de cuentas, depende 
de las elecciones y se mueve por la dinámica electoral... Entonces un compromiso que hemos tenido es darle más vida al partido en los tres años que no hay elecciones, entoes (sic) digamos que no solo sea en época de elecciones donde hay foros de diversos temas... donde hay entonces que implementar, digamos, otro tipo de espacios como cine foros... tejer más redes con otras agrupaciones, qué se yo la, Cruz Roja, las asociaciones de desarrollo, los grupos organizados del cantón... un reto importante es diversificar la agenda... Entonces, digamos, diversificar esa agenda, porque hay jóvenes que les interesa esa, la economía naranja, la cultura, ciencia y tecnología (pp. 37-38).

Así, se puede entender que, al menos, un sector de la juventud y demás integrantes del partido han iniciado un proceso de expansión de su práctica política en función de llegar a más segmentos de la población cuyos intereses exceden los temarios fijados en los tiempos y en las agendas electorales de las organizaciones. De esta manera, desde esta perspectiva, la política asume un carácter diferenciado de las campañas originadas para las votaciones nacionales o municipales, para ser un proceso de atención de preocupaciones y de temas de discusión nacional que no se restringen a dicha coyuntura.

En cuanto a las jóvenes entrevistadas del FA y del PT, la política, quizás, se encuentra más relacionada con la coordinación de los procesos de construcción de proyectos políticos en el campo electoral, para la primera. Para la segunda, se basa en la comunicación de estos durante el trabajo proselitista con otras personas de las comunidades. Siguiendo las declaraciones de ambas entrevistadas, las personas jóvenes participan de lleno en todas las fases de desarrollo de estos procesos partidarios, desde el planteamiento de los puntos centrales de los proyectos o programas políticos (su revisión o consolidación) hasta su divulgación y exposición.

\section{Discusión}

Como se ha detallado al inicio de este artículo, la recolección de los datos se realizó en medio del contexto del resultado de la primera y segunda rondas electorales, cuando el tema del pronunciamiento de la Comsión Interamericana de Derechos Humanos ( $\mathrm{CIDH})$ estaba aún mantenido por noticieros y re- 
des sociales. Sin embargo, salvo algunas referencias al margen por parte de las entrevistadas del PT y FA, el tema no fue mencionado por el resto del grupo de jóvenes candidatos.

En un sentido similar, otro tema que se destacó fue la coyuntura del referéndum del TLC. Solo tres personas jóvenes se refirieron a este (FA, PT y PAC) de forma directa y como un hito de participación clave dentro de la constitución de sus subjetividades políticas. Para ellas, este contexto fue clave en los niveles nacional e individual, y fue la puerta de entrada a su posterior involucramiento en organizaciones políticas. No obstante, este tema estuvo completamente ausente en los testimonios de los entrevistados del PUSC y del PLN; se señaló una única vez, en forma anecdótica, en las declaraciones de la joven candidata del ADC.

Al tener estos dos puntos en cuenta, se allana más el camino para la discusión de la noción de lo político y la percepción de la política con las cuales las personas jóvenes candidatas han narrado sus experiencias.

\section{Lo político}

De forma cotidiana, cuando se hace referencia a las nociones que poseen las personas sobre algo en particular, se infiere de inmediato que se trata de ideas generales, conocimientos laxos o representaciones elementales de ello. Sin embargo, estos suelen preceder o ser necesarias para el entendimiento de constructos, significados, procesos, ideas u otras elaboraciones más complejas o especializadas.

Esos elementos pudieron ser localizados en varias de las reflexiones realizadas por las personas jóvenes candidatas; principalmente, a partir de las posiciones que asumieron ante las diferentes estructuras relacionales con las que han interactuado en el transcurso de sus trayectorias de participación política.

Estas posturas, interpretadas en este artículo como un "yo" o un "nosotros" ante "otro" u "otros", no solo denotaban las manifestaciones más evidentes de sus subjetividades políticas, sino que sirvieron para connotar las dinámicas desplegadas por las personas jóvenes entrevistadas en sus contextos partidarios y políticos en general.

En este sentido, se destaca que la presencia de una unidad subjetiva en concordancia con lo adulto, además de ser una posición que reproduce el discurso y las estructuras adultocéntricas, se presenta, al parecer, en los espacios de participación política más afianzados o tradicionales dentro del contexto electoral.

Ese resultado confluye con los hallazgos de R. González-Sancho y Henríquez Cáceres (2016), quienes observaron que la reproducción de las prácticas y de los discursos adultos, por parte de las personas jóvenes candidatas a puestos de elección popular, se han desarrollado como un intento por superar o trascender los espacios de participación y las actividades a las que se les relega bajo la tutela de una persona adulta. Al mismo tiempo, este podría 
ser el caso de algunas de las narraciones analizadas en este artículo, en el sentido de que la obtención de candidaturas, al estar a tono con el partido, no representará un riesgo o una amenaza para este.

Entre tanto, las posiciones con mayor inclinación a la negociación-diálogo y a la confrontación-disputa se han presentado en aquellos espacios "menos tradicionales" (FA, PAC, PT, ADC). Esto podría deberse al rol diferenciado que, de acuerdo con sus trayectorias, el conjunto de candidatos ha tenido dentro de sus organizaciones en dinámicas que van desde la conformación de los cuadros de base de sus partidos (ADC, PT) hasta las labores propias de todo el proceso de desarrollo de las campañas electorales presidenciales y municipales (FA, PAC).

Así, se percibe que las posturas utilizadas por el grupo de jóvenes son formas diferenciadas de sortear, de adaptarse o de lidiar con las diferentes configuraciones de las estructuras partidarias y sus consiguientes manejos del poder organizativo y deliberativo. Este tipo de relaciones, con las cuales las personas jóvenes interactúan, están marcadas, especialmente, por el machismo y por el adultocentrismo, manifiestos en varias prácticas informales y formales de dichas instancias políticas.

En suma, al reproducir, negociar o disputar la participación y el protagonismo en los procesos políticos con lo adulto, lo joven no se limita orgánicamente a antagonizar con aquello, sino que parece mediar el conflicto en función de linderos a los cuales va siendo posible extenderse o reclamar su lugar en el contexto institucional. No obstante, ha de reconocerse que este proceso no se acaba en los límites de un partido político. Por el contrario, habría que observarlo en otros lugares y situaciones del quehacer sociopolítico.

\section{La política}

Respecto de las percepciones, siguiendo a Ferrater Mora (1964), se entiende que se forjan a partir de la aprehensión de nociones y de la experiencia misma del sujeto que aprehende una realidad, definiendo, en buena medida, su criterio o intelecciones sobre un contexto específico. Entonces, si las nociones son los elementos previos o necesarios para desarrollar el entendimiento sobre algo, las percepciones son los resultados de este proceso acerca de los diversos campos de experiencia.

Así, puede observarse que, si bien existen diferencias en las nociones de lo político en las personas entrevistadas, parecen haber pocas divergencias en las percepciones demostradas sobre la política, quizás, acusado por estar jugando en entornos con reglas, limitaciones y oportunidades muy similares dentro de los partidos en función de su condición de juventud. No obstante, se advierte un mayor agravio para las mujeres jóvenes entrevistadas por su condición de género.

En este orden de ideas, parece que las percepciones enunciadas de la política la definen como la "forma" en la que se ejecutan los procesos, el conjunto 
o las vías posibles de agencia de las acciones, así como la delimitación de ciertos rangos de relaciones sociales.

Como resultado, en primera instancia, el grupo de entrevistados connota la política como un medio para gestionar el desarrollo de determinadas propuestas dentro de las estructuras partidarias, mediante el diálogo, el convencimiento o la confrontación con las estructuras de dichas organizaciones.

También, ha cobrado cierta relevancia considerar como uno de los objetivos del quehacer político abordar las necesidades de la gente. Sin embargo, salvo un señalamiento de la entrevistada del FA, se acusa poca pericia en la interpretación o en las formas en las que se conocerían dichas urgencias y necesidades de las personas fuera del partido político.

En un tono similar, algunas de las personas jóvenes han señalado que el quehacer político debe tener una incidencia diferenciada, que no se limite al proceso electoral y que se direccione a llevar las discusiones de la agenda política a las comunidades.

En fin, parece que las diferencias en las percepciones explicitadas anteriormente están marcadas en los alcances y en los tiempos de permanencia de las acciones desplegadas, pero no tanto en las formas o aspectos que componen la política, como un medio de canalización de las urgencias de las personas.

\section{A modo de cierre}

La discusión sobre las especificidades y las interacciones entre los conceptos de lo político y la política, se vuelve más que necesaria cuando se tiene en cuenta que el conflicto o los conflictos que median en la construcción de lo social, de la sociedad y de la política, se convierten en un elemento nodal a la hora de entender los procedimientos, los procesos y las pugnas relacionales que determinan la visibilización, la priorización y la canalización de las necesidades o urgencias colectivas e individuales.

Dicho esfuerzo de comprensión podría clarificar qué posturas, actores, subjetividades o estructuras relacionales son las que determinan, como en el caso de los partidos políticos, las orientaciones, los alcances o las normativas que componen los acuerdos políticos que se construyen y se formalizan en los espectros formales e informales de la vida pública y política.

Finalmente, se debe considerar que el análisis presentado ubica el foco de atención dentro de los matices, de las contradicciones y de las coherencias de la praxis sociopolítica de jóvenes que actúan dentro de los linderos de partidos políticos. Por lo tanto, las reflexiones derivadas de lo político y de la política se limitan a una realidad formal bastante específica. Asimismo, valdrá la pena estudiar estas dimensiones conceptuales y fenomenológicas o indagar sobre sus particularidades o generalidades en instancias materiales más 
amplias, a fin de informar esas realidades que parecen escapar, muchas veces, al interés de las instituciones políticas, ante las cuales la ciudadanía joven, y de todas las edades, plantea sus reclamos, aspiraciones y descontentos.

\section{Bibliografía}

Abarca, Rocío. 2012. «Entre la participación y la voluntad política: La iniciativa popular y el caso del proyecto de ley gestión integral del recurso hídrico en Costa Rica». Anuario Centro de Investigación y Estudios Políticos 3: 48-79.

Aguilar López, Jesús. 2011. «Revisión del concepto de juventud y su relación con el mundo de la política». División de Derecho, Política y Gobierno, Universidad de Guanajuato, Campus Guanajuato. https://es.scribd.com/document/73044480/REVISION-DELCONCEPTO-DE-JUVENTUD-Y-SU-RELACION-CON-EL-MUNDODE-LA-POLITICA.

Alfaro, Josué. 2018. «Defensoría recibe reportes de violencia tras elecciones». CRhoy, el 14 de febrero de 2018, sec. Nacionales. https://www.crhoy.com/nacionales/defensoria-recibe-reportes-deviolencia-tras-elecciones/.

Alfaro, Josué, y Daniel Salazar. 2018. "Corte IDH: Costa Rica debe garantizar matrimonio igualitario». Semanario Universidad, el 9 de enero de 2018, sec. País. https://semanariouniversidad.com/pais/corte-idh-costa-rica-garantizarmatrimonio-igualitario/.

Arias-Cardona, Ana María, y Sara Victoria Alvarado. 2015. «Jóvenes y política: de la participación a la movilización formal». Revista Latinoamericana de Ciencias Sociales, Niñez y Juventud 13(2): 58194.

Arrieta, Esteban. 2018. «Nueva Asamblea Legislativa 'tendría' una visión más joven». La Republica.net, el 17 de abril de 2018, sec. ültima Hora. https://www.larepublica.net/noticia/nueva-asamblea-legislativa-tendriauna-vision-mas-joven.

Artavia Medrano, Argentina. 2018. Conformación de Asamblea legislativa en Elecciones presidenciales 2014 y 2018. 
Bendit, René, y Ana Miranda. 2017. «La nueva gramática de la juventud: un nuevo concepto en construcción». Última Década, núm. 17: 4-43.

Bourdieu, Pierre. 2002. "La 'juventud' no es más que una palabra». En Sociología y cultura, 163-73. Mexico: Grijalbo. https://periferiaactiva.files.wordpress.com/2016/03/bourdieu-lajuventud-no-es-mc3a1s-queuna-palabra.pdf.

Brito Lemus, Roberto. 1998. "Hacia una sociología de la juventud. Algunos elementos para la construcción de un nuevo paradigma de la juventud». Última Década 9. http://www.redalyc.org/pdf/195/19500909.pdf.

Cascante Lizano, Sharon. 2018. «Defensoría y UNA piden detener discursos de odio». La Prensa Libre, el 14 de febrero de 2018. http://www.laprensalibre.cr/Noticias/detalle/130699/defensoria-y-unapiden-detener-discursos-de-odio.

Chinchilla, Sofía, y Natasha Cambronero. 2018. "Corte Interamericana ordena abrir la puerta al matrimonio gay en Costa Rica». La Nación, el 9 de enero de 2018. https://www.nacion.com/el-pais/politica/corteinteramericana-notifica-a-costarica/LRJBJF6DWNHOFGBXSDNIVYSEDA/story/.

Cisterna Cabrera, Francisco. 2005. «Categorización y triangulación como procesos de validación del conocimiento en investigación cualitativa». Theoría 14 (1): 61-71.

Díaz Gómez, Álvaro. 2003. «Una discreta diferenciación entre la política y lo político y su incidencia sobre la educación en cuanto socialización política». Reflexión Política, 5 (9). http://www.redalyc.org/pdf/110/11000904.pdf.

Dussel, Enrique. 2016. 20 tesis de política. Cuarta reimpresión. México: Siglo $\mathrm{XXI}$.

Ferrater Mora, José. 1964. Diccionario de filosofía: Tomo II L - Z. 5. ${ }^{a}$ edición. Buenos Aires, Argentina: Editorial Sudamericana.

Gaínza Veloso, Álvaro. 2006. La entrevista en profundidad individual. En Canales, Manuel (2006) Metodologías de investigación social: Introducción a los oficios. Santiago, Chile: Lom Ediciones.

González-Sancho, Roy David. 2018. «Análisis de la trayectoria política en personas jóvenes candidatas a diputaciones en las elecciones 
nacionales de 2018» Ejecutivo 1. San Pedro, Montes de Oca: Centro Agenda Joven en Derechos y Ciudadanía.

González-Sancho, Roy, y Evita Henríquez Cáceres. 2016. «Participación juvenil en espacios formales de deliberación política: entre adultocentrismo y reproducción del discurso adulto» Revista Rupturas 7 (1): $125-147$.

Guanipa Pérez, Mary. 2011. "¿Cómo hacer un ensayo científico?». Revista Electrónica de Humanidades, Educación y Comunicación Social 3 (5): $1-10$.

Hidalgo-Capitán, Antonio Luis. 2012. El ensayo académico: Una guía para la elaboración de ensayos académicos en ciencias sociales. Huelva: Universidad de Huelva.

Jaramillo Ríos, Salud, y Víctor Mendoza Martínez. 2004. «Guía para la Elaboración de Ensayos de Investigación». Razón y Palabra Noviembre-octubre http://www.razonypalabra.org.mx/anteriores/n41/vmendoza.html.

Margulis, Mario, y Marcelo Urresti. 2008. La juventud es más que una palabra: ensayos sobre cultura y juventud. Buenos Aires, Argentina: Biblos.

Mouffe, Chantal. 2011. En torno a lo político. Segunda impresión. Buenos Aires: Fondo de Cultura Económica Argentina S.A.

Pérez Islas, José Antonio. 2000. Ser joven en méxico: concepto y contexto. En Jóvenes e instituciones en México, 1994-2000: actores, políticas y programas. Mexico: Instituto Mexicano de la Juventud. https://educiac.org.mx/pdf/Biblioteca/Juventud_e_Identidad/019SerJo ven_en_Mexico.pdf.

Pico Merchán, María Eugenia, y José Hoover Vanegas García. 2014. "Condición juvenil contemporánea: reflexiones frente a las realidades del actual contexto sociohistórico y laboral». Polis Revista Latinoamericana 39 (13). http://www.redalyc.org/articulo.oa? $\mathrm{id}=30533388018$.

Retamozo Benítez, Martín. 2009. «Lo político y la política: los sujetos políticos, conformación y disputa por el orden social». Revista Mexicana de Ciencias Políticas y Sociales LI (206): 69-91.

Sancho, Manuel. 2018. "Tras elecciones, ticos son menos sensibles hacia población LGBTI». CRhoy, el 7 de agosto de 2018, sec. 
Nacionales/política. https://www.crhoy.com/nacionales/traselecciones-ticos-son-menos-sensibles-hacia-poblacion-lgbti/.

Soto, Michael. 2018. "Juventud y rostro femenino: Asamblea intenta recuperar su imagen». CRhoy, el 2 de mayo de 2018, sec. Nacionales/ Política. https://www.crhoy.com/nacionales/juventud-yrostro-femenino-asamblea-intenta-recuperar-su-imagen/.

Taylor, S, y R Bogdan. 1987. Introducción a los métodos cualitativos de investigación: La búsqueda de significados. Barcelona, España: Paidós.

Villa Sepúlveda, María Eugenia. 2011. «Del concepto de juventud al de juventudes y al de lo juvenil». Revista Educación y Pedagogía, 23 (60): 147-57. 